\title{
Erratum: Numerical computations of the dynamics of fluidic membranes and vesicles [Phys. Rev. E 92, 052704 (2015)]
}

John W. Barrett, Harald Garcke, and Robert Nürnberg

(Received 14 November 2016; published 6 December 2016)

DOI: 10.1103/PhysRevE.94.069902

In our original submission, some numerical simulations used an incorrect sign convention for the outer normal, which also has an effect on one of our conclusions. In particular, the initial data for the discrete surfaces for the numerical experiments in Table I and Figs. 9, 21, 24, and 25 were created using a nonorientation preserving rotation. As a consequence, the discrete normals on $\Gamma^{h}(t)$ pointed into $\Omega_{-}^{h}(t)$, rather than into $\Omega_{+}^{h}(t)$, which means that the stated signs for $\bar{\varkappa}^{*}$ and $M_{0}^{*}$ were incorrect.

Upon correcting the normals for the discrete surfaces used in the simulations in Table I and Fig. 9, we obtain the results shown in Table I and Fig. 1 below, respectively. We observe that the effect of the alignment of the initial vesicle, which can be seen by comparing Table I and Fig. 1 below with Table 2 and Fig. 10 in the original article, respectively, is much less pronounced than initially thought.

Apart from correcting Table I and Fig. 9 as outlined above, the following corrections are needed for the paper.

(i) Page 10: In caption of Fig. 21, change $M_{0}^{*}=-33.5$ to $M_{0}^{*}=33.5$.

(ii) Page 10, right-hand column, 8 lines from the bottom: Remove "as well as on the orientation of the initial vesicle."

(iii) Page 11: In caption of Fig. 24, change $M_{0}^{*}=180$ to $M_{0}^{*}=-180$.

(iv) Page 12: In caption of Fig. 25, change $M_{0}^{*}=180$ to $M_{0}^{*}=-180$.

(v) Page 12, left-hand column, 9 lines from the top: Change $M_{0}^{*}=-33.5$ to $M_{0}^{*}=33.5$.

(vi) Page 12, right-hand column, 2 lines from the top: Change $M_{0}^{*}=180$ to $M_{0}^{*}=-180$.

(vii) Page 13, left-hand column, 18 lines from the top: Replace the text for point (3) with "The surface viscosity at which a transition between the different motions TT, TR, and TU occurs, strongly depends on the spontaneous curvature. In particular, we observed that for positive spontaneous curvature all transitions occurred for larger values of the surface viscosity. For negative spontaneous curvature, on the other hand, we observed that tumbling occurred already for much smaller values of the surface viscosity. Here we recall that our sign convention for curvature means that spheres have negative mean curvature".

TABLE I. Some inclination angles $\theta$ for the TT motions in Fig. 1.

\begin{tabular}{lccr}
\hline \hline$\mu_{\Gamma}^{*}$ & $\bar{\varkappa}^{*}=-5$ & $\bar{\varkappa}^{*}=0$ & $\bar{\varkappa}^{*}=5$ \\
\hline 0.05 & 0.198 & 0.178 & 0.179 \\
0.1 & 0.183 & 0.158 & 0.172 \\
0.2 & 0.144 & 0.116 & 0.163 \\
\hline \hline
\end{tabular}

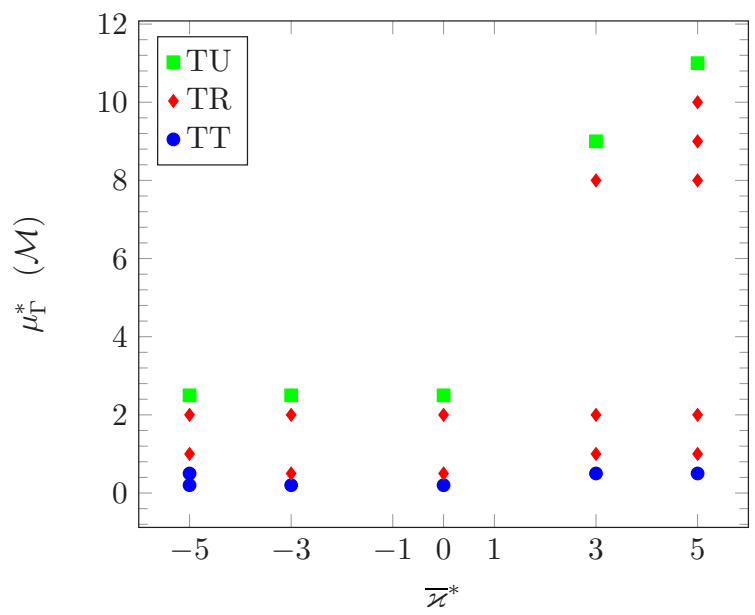

FIG. 1. Phase diagram for $\mathrm{Ca}=1 / \alpha^{*}=10$ for the domain $\bar{\Omega}=[-3,3]^{3}$, starting with biconcave shapes with $\mathcal{V}_{r}=0.8$ and the shortest axis in the $x_{1}$ direction. 